\title{
Trypanosoma brucei rhodesiense infection in a German traveller returning from the Masai Mara area, Kenya, January 2012
}

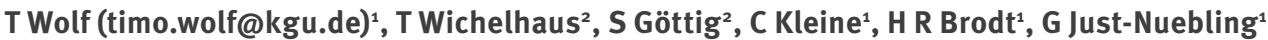

1. Department of Internal Medicine 2 - Infectious Diseases, Hospital of the J. W. Goethe University, Frankfurt, Germany

2. Institute of Medical Microbiology and Infection Control, Hospital of the J. W. Goethe University, Frankfurt, Germany

Wolf T, Wichelhaus T, Göttig S, Kleine C, Brodt HR, Just-Nuebling G. Trypanosoma brucei rhodesiense infection in a German traveller returning from the Masai Mara area, Kenya, January 2012 .

Euro Surveill. 2012;17(10):pii=20114. Available online: http://www.eurosurveillance.org/ViewArticle.aspx?Articleld=20114

In January 2012, a case of Human African Trypanosomiasis (HAT) has been identified in Germany in a traveller returning from the Masai Mara area in Kenya. The 62-year-old man had travelled to the Masai Mara game park from 18 to 19 January 2012 and developed fever on 28 January. The infection with Trypanosoma brucei rhodesiense was confirmed by laboratory testing three days hereafter.

\section{Case report}

On 28 January 2012, a 62-year-old man was hospitalised after a sudden onset of fever with temperature up to $39^{\circ} \mathrm{C}$ in a local hospital near Frankfurt, Germany. The fever started after his return from a holiday trip to Kenya from 8 to 28 January. Upon arrival in Germany and admittance to a local hospital, the patient was suspected to have malaria and treatment with Atovaquon / Proguanil was administered for two consecutive days. The diagnosis was made on the basis of a thin smear, which was later re-evaluated after the patient's transfer to the Infectious Diseases Department of Frankfurt University Hospital and no Plasmodium parasites were detected.

He had travelled by airplane directly from Frankfurt to Mombasa and back and spent all the time at a beach resort south of Mombasa except for a trip to the Masai Mara area from 18 to 19 January. For this trip, he flew from Mombasa to the OI Kiombo airstrip, stayed at a camp in the area, and then went on safari excursions within a radius of approximately $50 \mathrm{~km}$ from the camp. He wore shorts and short sleeved shirts most of the time and used insect repellents.

Despite anti-malarial treatment, the patient was still febrile on 31 January and was transferred to the Infectious Diseases Department of Frankfurt University Hospital. By then, the clinical symptoms had become more severe, with strong frontal headaches, vertigo, nausea and arthralgia. Fever was still high at $39.1^{\circ} \mathrm{C}$. He had two distinct, painless skin lesions over both tibiae (Figure 1), but no localised or disseminated lymph node enlargement.

Malaria parasites were not confirmed in Quantitative Buffy Coat, Giemsa-stained thin or thick blood smears and the malaria antigen test (BinaxNow) was negative. However, Trypanosoma brucei rhodesiense was detected in thick blood smears stained with Giemsa (Figure 2) on 1 February.

Treatment was started three hours after diagnosis of trypanosomiasis with $1 \mathrm{~g}$ of suramin as a continuous infusion over one hour. As the substance was not readily available, it was brought to Frankfurt University Hospital from the "Missionsärztliche Klinik" Würzburg, Germany, where a regular stock of suramin is kept. In parallel, the patient was given prednisolone to prevent

\section{FIGURE 1}

Chancres due to infection with Trypanosoma brucei rhodesiense in a German traveller returning from the Masai Mara area, Kenya, January 2012

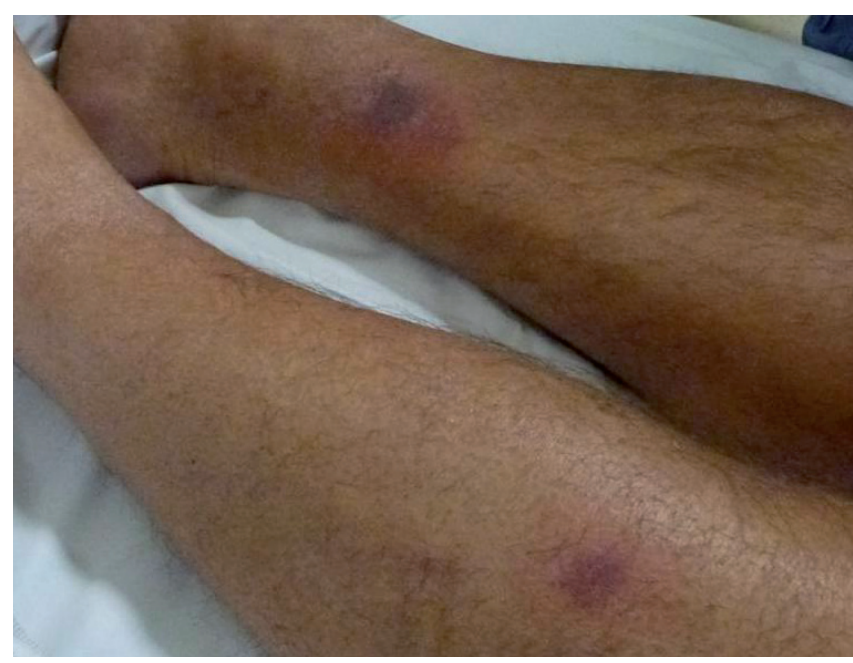


allergic reactions. The treatment was followed on day 1, 3, 7, 14 and 21 without complications.

A lumbar puncture performed on day 2 of therapy revealed a normal cerebrospinal fluid (CSF) pattern and a PCR with Trypanosoma spp. specific primers was negative from CSF as opposed to the peripheral blood, where it was found to be positive. The patient had leuko- and thrombopenia, an elevated complement regulatory protein (CRP) and aspartate and alanine transaminase levels two times the upper limit of normal. Electrocardiogram and echocardiography did not show any pathological findings.

The fever subsided on day 2 of treatment and no parasites were detected from day 3 of the treatment onwards. $T . b$. rhodesiense antibodies were detected by immunofluorescence testing performed at the reference laboratory (Bernhard Nocht Institute, Hamburg, Germany) on day 8 of treatment, 12 days after the first symptoms whilst having been negative on day 1 of treatment. The patient concluded his treatment as planned on day 21 without any residual problems and left the hospital.

\section{Discussion}

Following the detection of a case of Human African Trypanosomiasis (HAT) we screened the literature for recent alerts of HAT in Kenya and only ProMED had previously published a report on the occurrence of HAT in Kenya. This however, was documented almost 11 years before the current case $[1,2]$. About a month after the occurrence of the case described here, there was a further case of HAT from the Masai Mara area described in this issue of Eurosurveillance [3].

\section{FIGURE 2}

Giemsa-stained Trypanosoma brucei rhodesiense in a thick blood smear from a German traveller returning from the Masai Mara area, Kenya, January 2012

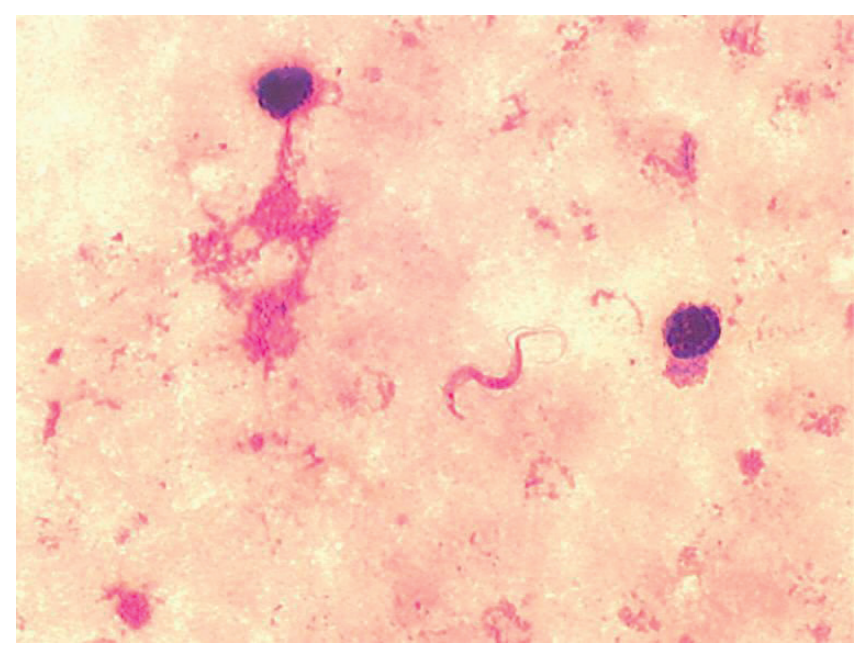

$64 x$ magnification
A literature research on PubMed revealed two publications that reviewed the epidemiology of HAT in nonendemic countries. A review of HAT cases imported into Europe between 2005 and 2009 included 11 cases, five of which were infected with $T$. $b$. rhodesiense. There were no cases described from Kenya, but two infected patients had travelled to the Serengeti, which directly borders Masai Mara [4]. In another report, the bibliographic data were supplemented by the World Health Organization (WHO) data on requests of antitrypanosomal drugs from hospitals in non-endemic countries treating travellers. These data showed that 94 cases of HAT were identified between 2000 and 2010, $72 \%$ of which were caused by T. b. rhodesiense. Although $59 \%$ of the cases were identified in Tanzania, with the vast majority of cases being tracked back to the Serengeti, no cases have been reported from Kenya [5].

Trypanosomiasis is a disease that occurs in local clusters, and one such cluster was identified in $\mathbf{2 0 0 2}$ through the TropNetEurop Sentinel Surveillance network when two index and seven consecutive cases were identified in non-disease endemic countries in Europe and South Africa [6]. These cases originated in the Serengeti and Tarangire National Parks in close proximity to Masai Mara, but with no documented case originating from the latter.

The above mentioned reports documented imported cases that were diagnosed in non-endemic countries. There are data on the cases diagnosed within the country however. The Kenyan reference hospital for sleeping sickness in Alupe, which is on the Ugandan border north of Lake Victoria, reported 31 patients with HAT caused by $T$. b. rhodesiense between 2000 and 2009. Twentytwo of the patients were diagnosed at the late stage of the diseases and coinfections and comorbidities were frequent [7]. Additionally, WHO extensively mapped the epidemiology of HAT in Africa between 2000 and 2009. For Kenya, sporadic cases were described in the very western provinces Bungoma, Teso and Busia, again on the Ugandan border, as well as in the Nyanza province. Epidemiological analysis of HAT in Kenya between 1950 and 2007 showed that infections occurred exclusively in these Western provinces, and the prevalence is altogether estimated to be low with only sporadic infections the 1990 s onwards $[8,9]$.

\section{Conclusion}

We identified a case of HAT due to $T$. $b$. rhodesiense infection in a traveller who had returned from the Masai Mara area, Kenya. After this case, another report of an imported HAT infection from this area was diagnosed one month later and communicated worldwide [10]. This is noteworthy, as there were no cases described from Masai Mara in the last decade. Previously, there was documented disease activity in Kenya which was limited to the western provinces, as well as Serengeti which is essentially in direct vicinity to Masai Mara. This report should alert clinicians to be aware of HAT when dealing with travellers from the area concerned. 
We have been in contact with WHO in Geneva, Switzerland, who confirmed that the local authorities in Kenya have been informed and a WHO team of experts has been sent to the area to elucidate the situation.

\section{Acknowledgments}

We would specifically like to thank Prof. Dr. med. August Stich, affiliated with the "Missionsärztliche Klinik Würzburg, Germany" for his generous and swift support in providing us with suramin. The authors would also like to thank all the medical staff and the diagnostic team involved in the treatment and diagnosis. We would like to extend our gratitude to the patient for agreeing to this publication.

\section{References}

1. ProMED-mail. Trypanosomiasis - Kenya. Archive Number: 20010511.0912. 11 May 2001. Available from: http://www. promedmail.org/direct.php?id=20010511.0912

2. ProMED-mail Trypanosomiasis, Human - Germany ex Kenya: Masai Mara. Archive Number: 20120202.1031130. 2 Feb 2012. Available from: http://www.promedmail.org/direct. php?id=20120202.1031130

3. Clerinx J, Vlieghe E, Asselman V, Van de Casteele S, Maes MB, Lejon V. Human African trypanosomiasis in a Belgian traveller returning from the Masai Mara area, Kenya, February 2012. Euro Surveill. 2012;17(10):pii=20111. Available from: http:// www.eurosurveillance.org/ViewArticle.aspx?Articleld=20111

4. Gautret $P$, Clerinx J, Caumes E, Simon F, Jensenius $M$, Loutan L, et al. Imported human African trypanosomiasis in Europe, 2005-2009. Euro Surveill. 2009;14(36):pii=19327. Available from: http://www.eurosurveillance.org/ViewArticle. aspx?Articleld $=19327$

5. Simarro PP, Franco JR, Cecchi G, Paone M, Diarra A, Ruiz Postigo JA, et al. Human African trypanosomiasis in nonendemic countries (2000-2010). J Travel Med. 2012;19(1):44-53

6. Jelinek T, Bisoffi Z, Bonazzi L, van Thiel P, Bronner U, de Frey A, et al. Cluster of African trypanosomiasis in travelers to Tanzanian national parks. Emerg Infect Dis. 2002;8(6):634-5.

7. Kagira JM, Maina N, Njenga J, Karanja SM, Karori SM, Ngotho JM. Prevalence and types of coinfections in sleeping sickness patients in Kenya (2000/2009). J Trop Med. 2011;2011:248914.

8. Rutto JJ, Karuga JW. Temporal and Spatial Epidemiology of Sleeping Sickness and use of geographical information System (GIS) in Kenya. J Vector Borne Dis. 2009;46(1):18-25.

9. Simarro PP, Cecchi G, Paone M, Franco JR, Diarra A, Ruiz JA, et al. The atlas of human African Trypanosomiasis: A contribution to global mapping of neglected tropical diseases. Int J Health Geogr. 2010;9:57.

10. ProMED-mail. Trypanosomiasis - Belgium ex Kenya: (Masai Mara). Archive Number: 20120222.1049305. 22 Feb 2012. Available from: http://www.promedmail.org/direct. php?id=20120222.1049305 